\title{
Linearization Techniques of Reservoir Simulation Equations - IMPES/IMPEM cases
}

\author{
FARKAS, Éva, MOL Plc Hungary
}

Paper presented at the Sth European Conference on the Mathematics of Oil Recovery, Leoben, Austria, 3-6 Sept.1996

\begin{abstract}
The main object of this paper is to examine and compare models based on the numerical background of linearization techniques. A new transformation procedure is introduced that helps to separate the nonlinearity of the flow equations from the nonlinearity of fluid calculations. This transformation procedure can help to decide an optimal technique when developing a new model. In addition, it clears the real and the apparent differences among the models, and it gives a possibility to arrive at a concise survey of the solution techniques. The detailed examination of the differences of IMPES and IMPEM techniques has revealed the origin of material balance error of the IMPES technique. The object-oriented C++ programming language was applied to the comparison studies. The basic $\mathrm{C}++$ classes and member functions are presented in the Appendix at the end of the paper.
\end{abstract}

\section{INTRODUCTION}

The reservoir simulators and numerical solution techniques of the model equations have developed greatly over the last four decades. In the early years, from the mid-fifties to the end of sixties, black oil modeling techniques were worked out. The direct sequential IMPES technique was developed in this period, and it has been widely used since then. All the coefficient functions of the governing equations of the IMPES model depend on pressure and saturations; thus the primary variables of this solution technique are the pressures and the saturations. The fluid characterization of black oil models depending only on pressure is simple, but suitable to simulate appropriately the conventional displacement technologies. Some problems, however, have arisen when applying the original IMPES method. Such problems are the correct description of the appearance or disappearance of phases, and modeling undersaturated systems when using only saturations as primary variables beside the pressure. To solve these problems the variable substitution is applied, e.g., $R_{s}$ is used as primary variable instead of $S_{g}$. Another problem is the material balance error. Many papers have dealt with the correct solution of these problems up to now.

The two pseudo hydrocarbon components of black oil models are unsuitable for describing processes in which the compositions of phases change greatly during displacement. Thus, from the early seventies, there has been a demand for the accurate simulation of enhanced oil recovery technologies using compositional and thermal models. In compositional cases the number of equations, and thus the unknown functions can be numerous, and the nonlinearity of governing equations is more severe than in black oil models. The mass (energy) conservation equations are often coupled with highly nonlinear algebraic systems. The coefficient functions of the governing differential equations, beside the pressure and saturations depend also on phase composition. In this case the number of conservation equations i.e. the number of components is greater than the number of phases. The well-proven practice of using black oil primary sets does not work. The saturations can easily be eliminated from the accumulation terms of the mass conservation equation of black oil models as the number of phases is equal to the number of components. In compositional cases, however, the elimination of the saturation and concentration terms from the accumulation term of the conservation equations is not a simple task. Many compositional and thermal models were developed in the seventies, but all models used an iteration technique, even in the IMPES-type case.

Aziz and Wong ${ }^{1}$ reviewed various models in detail. Based on the primary sets of these models (i.e. on the Jacobian variables) Aziz and Wong found two major categories of solution methods. One group of methods use pressure, saturation, (temperature), and phase compositions as primary variables. According to their study, this first group stems from a class of models where equilibrium $\mathrm{K}$ values are not composition-dependent. Another group using pressure, (temperature), and overall system composition - stems from a class of models in which 
compositional effects are important. They mentioned, however, that there has certainly been some overlap of techniques and processes.

There are many simplifications when formulating the governing differential equations. Generally, an important simplification of the models is the assumption of an instant and local thermody namic equilibrium. The solution techniques can be classified according to the calculation of local thermodynamic equilibrium. One group of models is referred to as the "coupled" models. In this type of models the equilibrium calculations are coupled with the flow calculations, and the fluid properties are consistent only at the end of the iteration process of flow calculation. Another type of model is known as the "decoupled" model. In the decoupled models the flow calculations are separated from the equilibrium calculations, and the primary sets are suitable to serve as input data for the pvT or EOS calculations.

The effectiveness of a solution technique essentially depends on two factors: (1) What is the "cost" of one iteration step i.e. the calculation of the Jacobian matrix ? (2) How "fast" is the convergence? In the following we examine these questions for 3P IMPES-type black oil models. Three primary sets are applied to simulate 1D water injection. The first primary set (iMMPEM) is an intensive type IMPEM set; this set is equivalent to the primary set of Young and Stephenson ${ }^{2}$. The second primary set is an IMPES one, and the third one is the extensive type IMPEM (eIMPEM) primary set of Ács $e t$. $a l^{3}$. Wong and $\mathrm{Aziz}^{4}$ have already compared the intensive and extensive type IMPEM models (Ref. 2. and Ref. 3.) for compositional cases. Our intention now is to compare the nonlinear behaviour of the solutions procedures when using: (1) saturations (compositions); (2) overall composition/densities; (3) extensive properties among the primary variables.

A $\mathrm{C}++$ program was developed for this comparison. The basic $\mathrm{C}++$ classes and member functions of this model are presented in the Appendix.

\section{LINEARIZATION OF THE MODEL EQUATIONS}

The discretized form of the model equation is

$$
\underline{\mathbf{F}}_{\mathrm{T}}\left(\underline{\mathbf{X}}_{\mathrm{T}}\right)=\mathbf{0}
$$

where the subscript $T$ indicates the total system, i.e. $\underline{\mathbf{X}}_{\mathbf{T}}$ denotes the vector of all thermodynamic properties of all the volume elements we intend to determine. The unknown vector $\underline{\mathbf{X}}_{\mathbf{T}}$ is obtained using an iteration procedure. The unknown variables can be divided into two parts. The first part is called the primary set, and is generally expressed by the Newton method from the flow equations and the saturation/volume constraint as the first step of the solution procedure. Then the additional or secondary variables are derived from the purely algebraic subsystem. Thus

$$
\underline{\mathbf{F}}_{\mathbf{T}}=\left\{\underline{\mathbf{E}}, \underline{\mathbf{E}}_{\mathbf{A}}\right\} \quad \text { and } \quad \underline{\mathbf{X}}_{\mathrm{T}}=\left\{\underline{\mathbf{X}}, \underline{\mathbf{X}}_{\mathbf{A}}\right\}
$$

can be written, where $F$ denotes the flow and saturation/volume constraint equations, while $\underline{\mathbf{X}}$ denotes the primary set. We assume, that

$$
\underline{\mathbf{X}}=\left\{\mathbf{X}_{1}, \ldots \mathbf{X}, \ldots, \mathbf{X}_{\mathrm{NE}}\right\},
$$

where NE is the number of volume elements. We examine the primary sets, our nonlinear system is

$$
E(X)=0 \ldots \ldots \ldots
$$

We name $\mathbf{X}$ the natural or basic variable set of the system, and assume

$$
X=\left(r_{1}, \ldots, r_{1}, \ldots, r_{N C}, p\right) \ldots \ldots
$$

where $r_{i}$ is the overall density of component $i$, and NC is the number of the components.

Let us select another primary set $\mathbf{Y}$. Because of the nonlinear nature of the problem generally $\mathbf{H}(\mathbf{X}, \mathbf{Y})=\mathbf{0}$. If $X$ can be explicitly expressed from the nonlinear system

$$
G(Y)=x \ldots \ldots
$$

and

$$
J_{G} \Delta_{l} \boldsymbol{Y}=\Delta, X \ldots \ldots
$$

can be written, where $\mathbf{J}_{\mathbf{G}}$ is the Jacobian matrix of the $\mathbf{G}$ transformation, and the subscript (superscript) 1 refers to the iteration level, and for example $\Delta_{1} \mathbf{X}=\mathbf{X}^{1+1}-\mathbf{X}^{1}$.

For all volume elements

$$
G(X)=Y \ldots \ldots \ldots
$$

We use the Newton method to derive $\underline{\mathbf{X}}$ from Eq. 1 ,

$$
\Delta X=-J_{E}^{-1} E(X) \ldots \ldots
$$

Eq. 4 can be substituted into Eq. 6 resulting in equation

$$
\Delta, Y=-J_{\underline{G}}^{-1} J_{E}^{-1} E(X) \ldots \ldots
$$

In Eq. (7) $\mathbf{J}_{\underline{F}}$ is the Jacobian matrix referring to the original system applying the basic variables. The $\mathbf{J}_{\mathbf{G}}$ blockdiagonal matrix is the Jacobian of the variable transformation. The convergence of the Newton method of the original problem depends on the nonlinearity of $\mathbf{J}_{\mathbf{F}}$, while the convergence of the Newton method when using the primary set $\underline{\mathbf{Y}}$ depends on the nonlinearity of matrix product $\mathbf{J}_{\mathbf{F}} \mathbf{J}_{\mathbf{G}}$. 


\section{P BLACK OIL EQUATIONS}

A 3P black-oil model is used to illustrate the above transformation. For simplicity, the nonlinearities of the accumulation and transport terms are assumed. The capillary-gravity expressions and rock compressibility are neglected. Only the gas component dissolution in the oil phase is assumed. The gas phase contains a gas component, the water phase contains a water component only. An IMPES-type technique is applied. Here afterwards, subscripts of capital letter $\mathrm{G}, \mathrm{O}$, and $\mathrm{W}$ refer to the components, and the subscripts of lower case letters $\mathrm{g}, \mathrm{o}$, and $\mathrm{w}$ refer to the phases.

Three primary sets were applied.

IIMPEM method: $\underline{\mathbf{X}}$ (Eq. 2) is an intensive type IMPEM set.

MMPES method: $\mathbf{Y}$ is the conventional primary set of a 3P black oil model

$$
\mathbf{Y}^{\mathrm{IMPES}}=\left(\mathrm{S}_{\mathrm{g}}, \mathrm{S}_{\mathbf{0}}, \mathrm{S}_{\mathrm{w}} \mathrm{p}\right),
$$

eIMPEM method: $\mathbf{Y}$ is the primary set of the original extensive type IMPEM VB model ${ }^{3}$.

$$
\mathbf{Y}^{\mathbf{e I M P E M}}=\left(\mathrm{m}_{\mathrm{G}}, \mathrm{m}_{\mathrm{O}}, \mathrm{m}_{\mathrm{W}}, \mathrm{p}\right\} .
$$

The phases and components and the primary set of the three techniques are shown in Table 1. The saturation/ volume constraint equations of the three models are summarized in Table 2.

In the following the difference equations of a volume element will be given for each of the three methods. We use subscript 0 to denote this general volume element, and subscript $\mathrm{k}(\mathrm{k}=1, \ldots, \mathrm{M})$ to denote its neighbors.

iIMPEM (intensive type IMPEM) method:

The mass conservation equations are

$$
\begin{aligned}
& F_{i}^{U M P E M}=V_{p} \Delta r_{i, 0}- \\
& \Delta t \sum_{k=1}^{M}\left[\frac{A K}{d}\right]_{k} \sum_{j=1}^{N P}\left(\frac{k_{j}}{\mu_{j}} C_{k_{j}}\right)^{u} \rho_{j}\left(p_{k}^{n+1}-p_{0}^{n+1}\right)- \\
& \Delta t Q_{h 0}^{n}=0, \quad i=G, O, W
\end{aligned}
$$

We can concisely write the above equations as

$$
\begin{gathered}
F_{i}^{u M P Q M}=V_{p} \Delta r_{i, 0}- \\
\Delta t \sum_{k=1}^{M} T_{i, k}\left(p_{k}^{n+1}-p_{0}^{n+1}\right)-\Delta t Q_{t, 0}^{n}=0, \ldots \\
i=G, O, W
\end{gathered}
$$

The saturation constraint equation is:

$$
\begin{aligned}
F_{4}^{\text {UMPEN }}= & \frac{r_{g}}{\rho_{g}}+\frac{r_{0}}{\rho_{o}}+\frac{r_{W}}{\rho_{w}}-1= \\
& \frac{B_{g}}{\rho_{G}^{s d d}} r_{G}+\frac{B_{0}-R_{s} B_{g}}{\rho_{0}^{s d d}} r_{G}+\frac{B_{w}}{\rho_{W}^{s d d}} r_{W}-1=0
\end{aligned}
$$

IMPES method:

The component conservation equations are:

$$
\begin{aligned}
F_{G}^{\text {MPPES }}= & V_{p}\left(\frac{S_{g}}{B_{g}} \rho_{G}^{S t d t}+\frac{S_{o} R_{s}}{B_{o}} \rho_{o}^{s t h}\right)- \\
& \Delta t \sum_{k=1}^{M} T_{G, k}\left(p_{k}^{n+1}-p_{0}^{n+1}\right)-\Delta t Q_{G, 0}^{n}=0,
\end{aligned}
$$

$$
\begin{aligned}
F_{O}^{n A P E S}= & V_{p} \frac{S_{0}}{B_{0}} p_{O}^{s d d}- \\
& \Delta t \sum_{k=1}^{M} T_{O, k}\left(p_{k}^{n+1}-p_{0}^{n+1}\right)-\Delta t Q_{o, 0}^{n}=0,
\end{aligned}
$$

$$
\begin{aligned}
F_{W}^{n M P S S}= & V_{p} \frac{S_{w}}{B_{w}} \rho_{W}^{s t d}- \\
& \Delta t \sum_{k=1}^{M} r_{W, k}\left(p_{k}^{n+1}-p_{0}^{n+1}\right)-\Delta t Q_{W, 0}^{n}=0,
\end{aligned}
$$

The saturation constraint equation is:

$$
F_{4}^{I M P E S}=S_{g}+S_{o}+S_{w}-1=0 \ldots
$$

eIMPEM (extensive type) method:

The component conservation equations are:

$$
\begin{gathered}
F_{i}^{\text {eIMPES }}=\Delta m_{b, 0}- \\
\Delta t \sum_{k=1}^{M} T_{i, k}\left(\varphi_{k}^{n+1}-p_{0}^{n+1}\right)-\Delta t Q_{i, 0}^{n}=0, . \\
\quad i=G, O, W
\end{gathered}
$$

The volume constraint equation is:

$$
F_{4}^{e I M P E M}=V_{f}-V_{p}=0 \ldots \ldots
$$

A diagonal block of each Jacobian matrix can be seen in Fig. 1. - Fig. 3. Due to the IMPES-type handling of component balance equations, the linearization of the flow terms result in the same terms of the Jacobian matrices for the three methods. An off-diagonal block of the Jacobians is shown in Fig. 4. 
We can explicitly express $\mathbf{X}$ when transforming the iIMPEM primary variables to IMPES ones.

$$
G\left(Y_{\text {MMPS }}\right)=X
$$

A Jacobian block of this transformation is shown in Fig 5. If we right multiply the Jacobian matrix $J_{\mathrm{F}}$ of the iIMPEM method (Fig 1) with the Jacobian matrix $\mathbf{J}_{\mathbf{G}}$ of the $\mathbf{G}$ transformation (Fig. 5) we obtain the Jacobian matrix of the IMPES method (Fig 2).

Similarly, if we right multiply the Jacobian matrix of the IMPES method with the inverse Jacobian of the $\mathbf{G}$ transformation, we obtain the Jacobian matrix of the iIMPEM method. Here, it is very important to note that $\Delta \mathbf{X}$ (masses) can always transform to $\Delta \mathbf{Y}_{\text {IMPES }}$ (saturation). The transformation $\Delta \mathbf{Y}_{\text {IMPES }}$ set to $\Delta \mathbf{X}$ set is valid, however, only in three phase cases.

As for the IMPEM primary sets, there are differences not only because

$$
m_{i}=V_{p} r_{i} \quad \text { or } \quad m_{i}=V_{f} r_{i}, \quad i=G, O, W,
$$

but also because the iIMPEM model uses saturation constraint, and the eIMPEM model uses volume constraint equations.

\section{SIMULATION RESULTS}

The boundary conditions of the ID simulation were: constant rate water injection at $\mathrm{x}=0$, while the original pressure was maintained at the $x=L$, where $L$ was 1000 $\mathrm{m}$. The number of the grid block was 80 . Initial pressure was $192 \mathrm{bar}$, and initial saturations were $\mathrm{S}_{\mathrm{gi}}=.1, \mathrm{~S}_{\mathrm{oi}}=6$, $S_{\mathrm{wi}}=.3$. The initial hydrocarbon pore volume was $450 \mathrm{~m}^{3}$ $\mathrm{S}_{\mathrm{gr}}=.05, \mathrm{~S}_{\mathrm{oi}}=.25$, and water injection rate was $1 \mathrm{~m}^{3} /$ day. The results on Figs. $7-12$. refer to 270 days when the injected water was $60 \%$ of the initial hydrocarbon PV.

Figs. 7, 9. and 11. show results without iterations. Figs. 8., 10., and 12. show results with one iteration. One can see, that only the IMPES results are different in Figs. 7, 9 , and 11. After one iteration the three techniques give the same solutions. Nevertheless, only the results of IMPES calculations were different after the iteration; the two IMPEM models gave practically the same results as those resulted in the first step. The results of the IMPEM calculations are practically the same, and both methods worked without material balance errors. Yet the two solution techniques differ. The iIMPEM primary variables are dependent; as for isothermal cases, the number of independent properties is only NC. The saturation and volume constraint was not satisfied exactly. However, in this simple example the errors were negligible. The primary variables of the eIMPEM method are independent. During the simulation process the saturation constraints were satisfied, but very small volume balance errors appeared and these were corrected in the subsequent timesteps. The IMPES calculation resulted in material balance errors the saturation and volume constraint equations were satisfied. The above statements are summarised in Table 3.

\section{TRANSFORMATION OF THE PRIMARY SETS}

The Jacobian matrix of the IMPES technique from Eq. (7)

$$
J_{\text {LMPES }}=J_{F} J_{G} \ldots \ldots \ldots
$$

We can calculate $\Delta \mathbf{X}$ from Eq. 4. The pressure is invariable when applying this transformation and we can get correct masses of the iIMPEM technique, and a corrected saturation set can be calculated from the new masses. After this transformation the saturation and volume constraint equations are no longer satisfied exactly in the first step, as we switch to the IIMPEM method. With this transformation we get the same results as the iIMPEM methods in the first step. (See IMPES_to_IMPEM solution on Figs. 7, 9, and 11.) The additional work is only the back transformation of the primary set by volume elements (Fig. 5). We can give a similar transformation for two phase cases (Fig. 6).

Starting from the iIMPEM Jacobian matrix and transforming $\mathbf{Y}_{\text {IMPES }}$ to $\mathbf{X}$ after the solution of the system of linear equations, we have the IMPES solution with the material balance errors for one-step solutions.

Summarizing the above, we can obtain iIMPEM results starting from iIMPEM Jacobian matrix or starting from IMPES Jacobian matrix and transforming back the primary set at the end of the IMPES step. The pressures are the same for both cases but the techniques are differ with regard to how the saturations (concentrations) are calculated, i.e. whether we calculate $r_{i}$ from $S_{j}$ $(i=G, O, W, j=g, o, w)$, or $S_{j}$ from $r_{i}$

\section{DISCUSSION}

The primary set selection of IMPES models has a primarily traditional cause. The problems of IMPES technique come from the original transformation of basic primary set.

The iIMPEM and eIMPEM methods are more effective than the IMPES method, even in black oil case. The calculation of one iteration step requires a similar computing time for all three cases, but the convergence of IMPEM methods is faster than that of the IMPES method. The fluid properties of the ilMPEM method are consistent only at the end of the iteration process of a timestep. However, sometimes one can get the result without iterations. The fluid properties of the eIMPEM method are always consistent, but numerical errors appear in the 
volume balance errors. The timesteps of the eIMPEM techniqe are chosen in such a way that the volume balance errors should be kept small enough, and the small volume balance errors are corrected in the subsequent timestep.

A transformation technique is introduced to switch one simulation technique to another. One can improve the results of existing IMPES model based on this transformation technique. This modification can be realized by volume elements after the solution of an IMPES timestep. According to our examinations the IIMPEM and IMPES techniques resulted in the same pressure distribution. Thus, another improvement of IMPES technique can be realized, if we use only the the pressure of IMPES solution and calculate the component mass changes from the mass balance equations as, in the eIMPEM method. This technique can be convenient for compositional cases, when the Jacobian matrix of the primary set transformation $\mathbf{J}_{\mathbf{G}}$ can be very complicated. More recently, a similar technique has been applied by Coats et. al. ${ }^{5}$ in their fully implicit compositional model.

The examination of fully implicit models is the next task to be carried out. Then, we can solve a more nonlinear system than in the IMPES/IMPEM cases. The transmissibility derivatives will modify the structure and of the Jacobian matrix, too.

\section{THE COMPUTER MODEL}

A $\mathrm{C}++$ model has been developed for this comparison. The main classes, together with the member functions, are presented in the Appendix.

\section{CONCLUSION}

We compare the solution techniques when using "saturation/phase composition" and "overall concentration/ density"-type primary sets. Our results, and the good experiences of the last decade using IMPEM models have proved the superiority of "overall concentration/density"type primary sets in every respect.

The examinations of this paper have cleared up the origin of the material balance errors of the IMPES method. If we consider the nonlinear coupling of Eq. 17, and calculate the component masses from phase saturations applying Eq. 4 (see Fig. 5) there will be no material balance errors. By using this correction, however, we switch from the IMPES technique to an IMPEM one.

The transformation technique presented in this paper can be applied to examine other solution methods, and it may help to develop an optimal linearization technique for reservoir simulators.

\section{NOMENCLATURES}

A area, $\mathrm{m}^{2}$

B formation volume factor, $\mathrm{m}^{3} / \mathrm{m}^{3}$

C mass fraction, $\mathrm{kg} / \mathrm{kg}$

$\mathrm{d}$ distance between grid points, $\mathrm{m}$

$\mathrm{k}$ relative permeability

$\mathrm{K}$ absolute permeability, $\mathrm{m}^{2}$

L transmissibilities (mass rates, $\mathrm{kg} / \mathrm{s} . \mathrm{Pa}$ )

m mass, $\mathrm{kg}$

$\mathrm{M}$ the number of neighboring elements

$\mathrm{NC}$ the number of components

$\mathrm{NE}$ the number of volume elements

NJ the number of Jacobian variables

NP the number of phases

r overall component density, $\mathrm{kg} / \mathrm{m}^{3}$

Rs dissolved gas--oil ratio, $\mathrm{m}^{3} / \mathrm{m}^{3}$

Q mass source, $\mathrm{kg} / \mathrm{s}$

$t$ time, $s$

$\mathrm{V}$ volume, $\mathrm{m}^{3}$

$\mu$ viscosity Pa.s

$\rho$ density, $\mathrm{kg} / \mathrm{m}^{3}$

\section{Subscripts}

0 general volume element

f fluid

G gas component

g gas phase

i component, $i=1, \ldots, N C$

j phases, $\mathrm{j}=1, \ldots, \mathrm{NP}$

k neighboring volume element, $\mathrm{k}=1, \ldots, \mathrm{M}$

O oil component

- oil phase

$p$ pore

W water component

w water phase

\section{Superscripts}

1 iteration

u upstream

n old time level

$n+1$ new time level

\section{REFERENCES}

1 Aziz, K. and Wong, T.W.:"Considerations in the Developments of Multipurpose Reservoir Simulation Models", Proceedings of the First and Second International Forum on Reservoir Simulation, Alpbach, Sept. 12-16, 1988 and Sept. 4-8. 1989

2 Young, L.C. and Stephenson, R.E.: "A Generalized Compositional Approach for Reservoir Simulation", SPEJ, Oct., 1983, (727-742)

3 Ács, G, Doleschall S., and Farkas, É.:"General Purpose Compositional Model", SPEJ, Aug. 1985 (543-553) 
4 Wong, T.W., Firoozabadi, A., and Aziz, K.: "Relationship of the Volume-Balance Method of Compositional Simulation to the Newton-Raphson Method", SPERE Aug. 1990, (415-422)

5 Coats, K.H., Thomas, L.K. and Pierson, R.G.: "Compositional and Black Oil Reservoir Simulation", Paper SPE 29111 presented at the 13th Symposium on Reservoir Simulation, San Antonio, TX, Feb. 1995

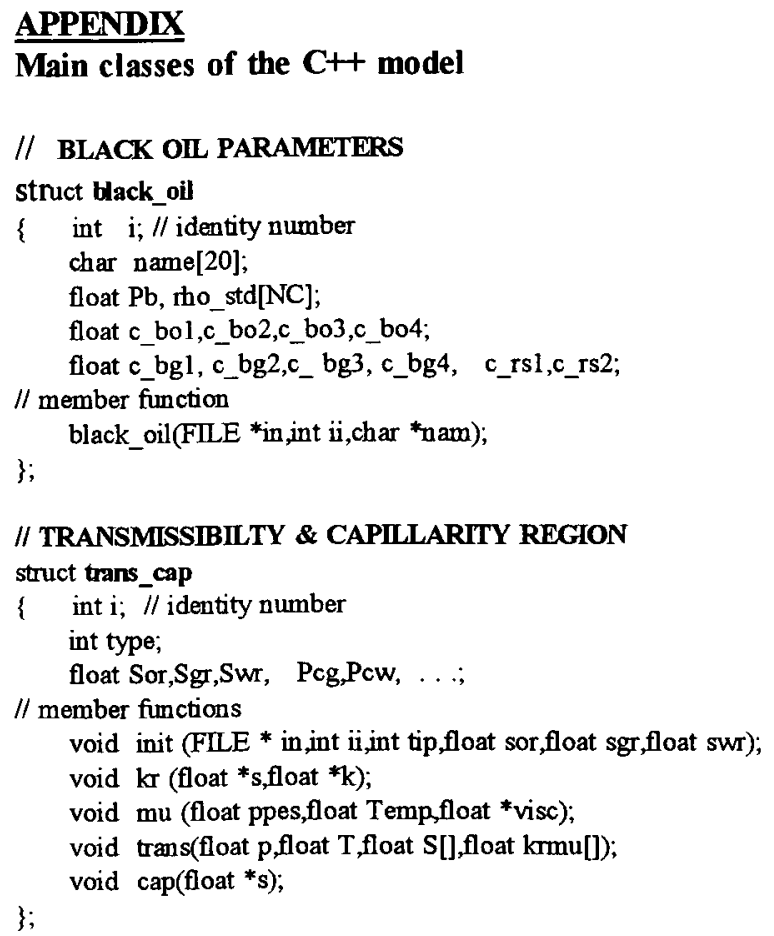

\section{// ROCK PROPERTIES}

struct rock

$\{$ int ie; // identity number

float phii,phi; // porosity

float pi,cr, V;

float $\mathrm{dVp} ; \quad / / \partial \mathrm{Vp} / \partial \mathrm{p}$

$/ /$ member functions

void init(int $i$,float ff,float $c$ );

void init2(float pi,float Vpi,FILE *,int);

\}

\section{// FLUID SYSTEM}

struct fluid

\{ int ie; // identity number

int type;

black_oil *b; // connect black oil parameters

trans_cap *tc; // connect trans-cap region

// primary

float $\mathrm{p}, \mathrm{T}, \mathrm{m}[\mathrm{NC}], \mathrm{r}[\mathrm{NC}]$;

I/ secondary

float Vf, C $[N C][N P]$, ro[NP], S[NP];

// black_oil

float bo,bg,bw, rs;

float bop,bgp,bwp,rsp;

// transmissibilities

float kmmu[NP];

// changes

float dpt, dme[NC], dm[NC], dr[NC], dS[NP];

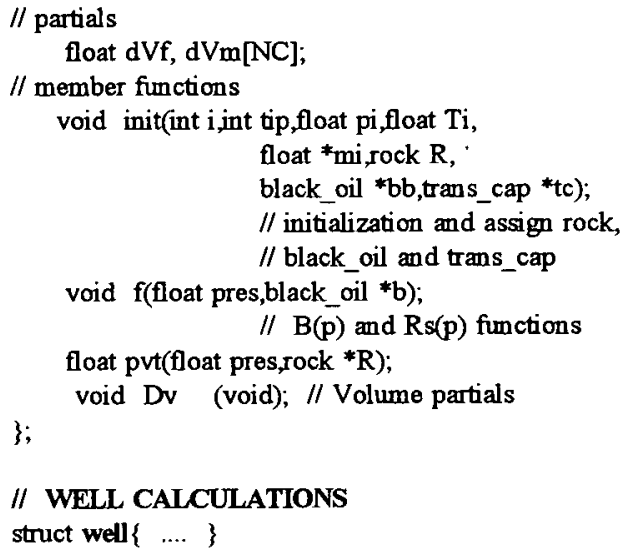

\section{// WELL CALCULATIONS}

struct well $\{\quad \ldots$.

\section{// A JACOBIAN BLOCK}

struct JB

( float J[NJ][NJ]; // J[row][column]

void clear(float ii);

3

\section{// A BOUNDARY SURFACE}

struct boundary

\& int $\mathrm{ib}$; // identity number

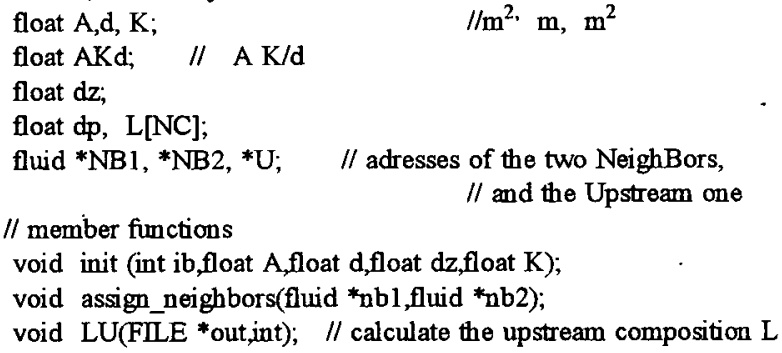

\section{// A VOLUME ELEMENT} struct volume_element

$\{$ int ie; // identity number

float $\mathrm{x}, \mathrm{y}, \mathrm{z} ;$ // grid point

float $\mathrm{rv}$; //volutmetric error

well *W; // well connention if $W \neq N U L L$

fluid ${ }^{*} \mathrm{~F} ; \quad$ // the adress of fluid belonging to this block

rock * R; // the adress of rock belonging to this block

int $\quad \mathrm{M}$; / the number of neighbors

fluid ${ }^{* *} \mathrm{~N} ; \quad / /$ adresses of fluids of neighboring blocks

boundary ${ }^{* *} \mathrm{~B} ; \quad / /$ adresses of boundary surfaces

JB *JD; // diagonal Jacobian block

JB **J; // nondiagonal Jacobian blocks

JB G, G_INV,

float residual[NJ];

float X[NJ]; // primary set

float deltaX[NJ;

float $\mathrm{Y}[\mathrm{NJ}] ;$ // transformed primary, set

// member functions

void connectFRNB(void); // initialization of connections

void clear_dme(void);

void clear_dm_dpt(void);

void qchang (void); // well calculations

void coeffg (FILE *out,int wri); // generate the Jacobian blocks void new_dm_dpt (FILE* out,int wi);

void get_G(FILE *out,int wri); // generate tranformation block \} void get_G_INV(FILE *out,int wri);

enum techniques IIIMPEM, from IIMPEM to IMPES, IMPES, from_IMPES_to _iIMPEM, OIMPEM\}; 
Table 1. The masses of components and phases, and the primary variables of the three methods

\begin{tabular}{|c|c|c|c|c|c|c|}
\hline \multirow{2}{*}{$\begin{array}{l}\text { Comp- } \\
\text { onents }\end{array}$} & \multicolumn{3}{|c|}{ Phases } & \multicolumn{3}{|c|}{ Primary vaniables } \\
\hline & $\begin{array}{c}\text { gas } \\
\mathrm{g}\end{array}$ & $\begin{array}{c}\text { oil } \\
0\end{array}$ & $\begin{array}{c}\text { water } \\
\text { w }\end{array}$ & $\begin{array}{l}\text { iIMPEM } \\
\text { method }\end{array}$ & $\begin{array}{l}\text { IMPES } \\
\text { method }\end{array}$ & $\begin{array}{l}\text { eIMPEM } \\
\text { method }\end{array}$ \\
\hline $\begin{array}{c}\text { Gas } \\
\mathrm{G}\end{array}$ & $\mathrm{m}_{\mathrm{G}, \mathrm{g}}$ & $\mathrm{m}_{\mathrm{G}, \mathrm{o}}$ & & $\begin{array}{c}r_{G}= \\
\left(m_{G, g}+m_{G, o}\right) / V_{p}\end{array}$ & $\mathrm{~S}_{\mathrm{g}}$ & $\begin{array}{c}\mathrm{m}_{\mathrm{G}}= \\
\mathrm{m}_{\mathrm{G}, \mathrm{g}}+\mathrm{m}_{\mathrm{G}, \mathrm{o}}\end{array}$ \\
\hline $\begin{array}{c}\text { Oil } \\
0\end{array}$ & & $\mathrm{~m}_{\mathrm{O}, \mathrm{o}}$ & & $\begin{array}{c}\mathrm{r}_{\mathrm{O}}= \\
\mathrm{m}_{\mathrm{O}, 0} / \mathrm{V}_{\mathrm{p}}\end{array}$ & $\mathrm{S}_{\mathrm{o}}$ & $\begin{array}{l}\mathrm{m}_{\mathrm{O}}= \\
\mathrm{m}_{\mathrm{O}, \mathrm{o}}\end{array}$ \\
\hline \multirow[t]{2}{*}{$\begin{array}{c}\text { Water } \\
\text { W }\end{array}$} & & & $\mathrm{m}_{\mathrm{w}, \mathbf{w}}$ & $\begin{array}{c}\mathrm{r}_{\mathrm{W}}= \\
\mathrm{m}_{\mathrm{W}, \mathrm{w}} \mathrm{N}_{\mathrm{p}}\end{array}$ & $S_{w}$ & $\begin{array}{l}\mathrm{m}_{\mathrm{W}}= \\
\mathrm{m}_{\mathrm{W}, \mathrm{w}}\end{array}$ \\
\hline & $\begin{array}{c}r_{g}= \\
m_{G, g} / V_{p}\end{array}$ & $\begin{array}{c}r_{o}= \\
\left(m_{G, o}+m_{O, o}\right) / V_{p}\end{array}$ & $\begin{array}{c}\mathrm{r}_{\mathrm{w}}= \\
\mathrm{m}_{\mathrm{W}, \mathrm{w}} / \mathrm{V}_{\mathrm{p}}\end{array}$ & $\mathrm{p}$ & $\mathbf{p}$ & $\mathrm{p}$ \\
\hline
\end{tabular}

Table 2. Saturation/volume constraint equations of the methods

\begin{tabular}{|c|c|}
\hline \hline Method & Constraint equation \\
\hline \hline iIMPEM & $\mathrm{r}_{\mathrm{g}} / \rho_{\mathrm{g}}+\mathrm{r}_{\mathrm{o}} / \rho_{\mathrm{o}}+\mathrm{r}_{\mathrm{w}} / \rho_{\mathrm{w}}-1=0$ \\
\hline IMPES & $\mathrm{S}_{\mathrm{g}}+\mathrm{S}_{\mathrm{o}}+\mathrm{S}_{\mathrm{w}}-1=0$ \\
\hline eIMPEM & $\mathrm{V}_{\mathrm{f}}-\mathrm{V}_{\mathrm{p}}=0$ \\
\hline
\end{tabular}

Table 3. Some features of the methods when calculating without iteration

\begin{tabular}{|c|c|c|c|c||}
\hline Method & $\begin{array}{c}\text { Material } \\
\text { balance } \\
\text { enors }\end{array}$ & $\begin{array}{c}\text { Saturation } \\
\text { constraint } \\
\text { errors }\end{array}$ & $\begin{array}{c}\text { Volume } \\
\text { constraint } \\
\text { enors }\end{array}$ & $\begin{array}{c}\text { Variable } \\
\text { substitution } \\
\text { is needed } \\
\text { when gas or oil phases } \\
\text { appeartdisappear }\end{array}$ \\
\hline ilMPEM & no & yes & yes & no \\
\hline IMPES & yes & no & no & yes \\
\hline eIMPEM & no & no & yes & no \\
\hline
\end{tabular}




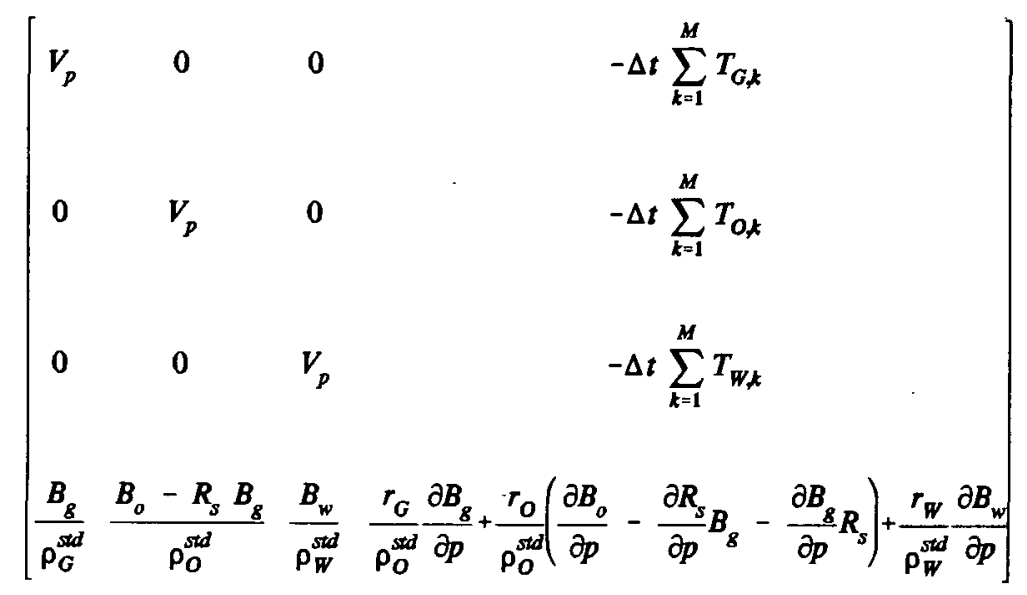

Figure 1. IIMPEM method: a diagonal block of Jacobian matrix

$$
\left[\begin{array}{cccc}
V_{p} \frac{\rho_{G}^{s s d}}{B_{g}} & V_{p} \frac{\rho_{G}^{s s d}}{B_{o}} R_{s} & 0 & V p\left[\left(\frac{\partial R_{s}}{\partial p} B_{o}-\frac{\partial B_{o}}{\partial p} R_{s}\right) \frac{S_{o}}{B_{o}^{2}}-\frac{\partial B_{g}}{\partial p} \frac{S_{g}}{B_{g}^{2}}\right] \rho_{G}^{s s d}-\Delta t \sum_{k=1}^{M} T_{o, k} \\
0 & V_{p} \frac{\rho_{o}^{s s d}}{B_{o}} & 0 & -V_{p} \frac{\partial B_{o}}{\partial p} \frac{S_{o} \rho_{o}^{s d d}}{B_{o}^{2}}-\Delta t \sum_{k=1}^{M} T_{o, k} \\
0 & 0 & V_{p} \frac{\rho_{W}^{s t d}}{B_{w}} & -V_{p} \frac{\partial B_{w}}{\partial p} \frac{S_{w} \rho_{W}^{s t d}}{B_{w}^{2}}-\Delta t \sum_{k=1}^{M} T_{W, k} \\
1 & 1 & 1 & 0
\end{array}\right]
$$

Figure 2. IMPES method: a diagonal block of Jacobian matrix

$$
\left[\begin{array}{cccc}
1 & 0 & 0 & -\Delta t \sum_{k=1}^{M} T_{G, k} \\
0 & 1 & 0 & -\Delta t \sum_{k=1}^{M} T_{o, k} \\
0 & 0 & 1 & -\Delta t \sum_{k=1}^{M} T_{W, t} \\
\frac{B_{g}}{\rho_{G}^{s d d}} \frac{B_{o}-R_{s} B_{g}}{\rho_{G}^{s d d}} \frac{B_{w}}{\rho_{W}^{s d d}} \frac{m_{G}}{\rho_{G}^{s d d}} \frac{\partial B_{g}}{\partial p}+\frac{m_{o}}{\rho_{o}^{s d d}}\left(\frac{\partial B_{o}}{\partial p}-\frac{\partial R_{s}}{\partial p} B_{g}-\frac{\partial B_{g}}{\partial p} R_{s}\right)+\frac{m_{W}}{\rho_{W}^{s d}} \frac{\partial B_{W}}{\partial p}
\end{array}\right]
$$

Figure 3. eIMPEM method: a diagonal block of Jacobian matrix 
$J_{k}=\left[\begin{array}{cccc}0 & 0 & 0 & -\Delta t T_{G, k} \\ 0 & 0 & 0 & -\Delta t T_{O, k} \\ 0 & 0 & 0 & -\Delta t T_{W, k} \\ 0 & 0 & 0 & 0\end{array}\right]$

Figure 4. An off-diagonal block of Jacobian for IIMPEM, IMPES, and EIMPEM

methods; $1 D$ case $k=1$ or 2 .

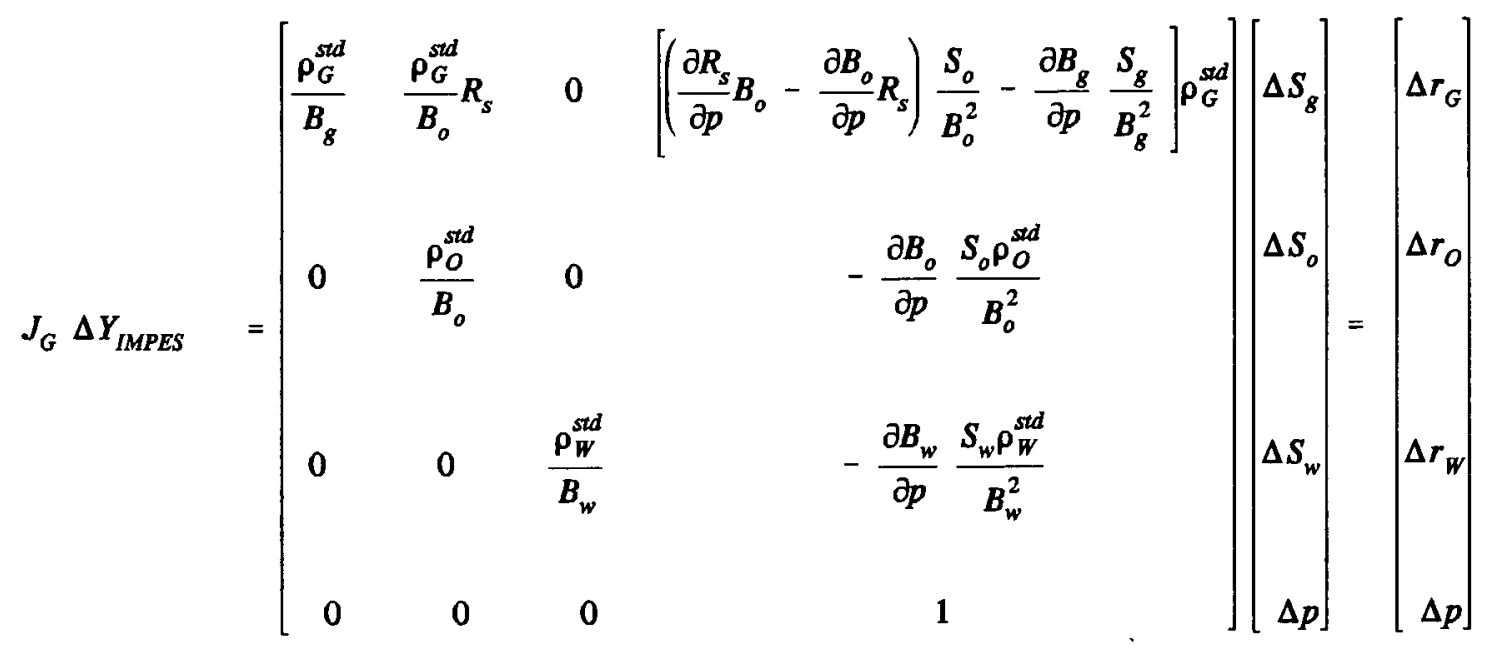

Figure 5. A Jacobian block of IMPES transfonmation; 3P system

$$
J_{G} \Delta Y_{I M P E S-2 P}=\left[\begin{array}{cccc}
\frac{\rho_{G}^{s t d}}{B_{o}} S_{o} & \frac{\rho_{G}^{s t d}}{B_{o}} R_{s} & 0 & \left(\frac{\partial R_{s}}{\partial p} B_{o}-\frac{\partial B_{o}}{\partial p} R_{s}\right) \frac{S_{o} \rho_{G}^{s d}}{B_{o}^{2}} \\
0 & \frac{\rho_{o}^{s t d}}{B_{o}} & 0 & -\frac{\partial B_{o}}{\partial \dot{p}} \frac{S_{o} \rho_{o}^{s t d}}{B_{o}^{2}} \\
0 & 0 & \frac{\rho_{W}^{s t d}}{B_{w}} & -\frac{\partial B_{w}}{\partial p} \frac{S_{w} \rho_{W}^{s t d}}{B_{w}^{2}} \\
0 & 0 & 0 & 1
\end{array}\right]\left[\begin{array}{c}
\Delta R_{s} \\
\Delta S_{w} \\
\Delta p
\end{array}\right]=\left[\begin{array}{c}
\Delta r_{G} \\
\Delta r_{o} \\
\Delta r_{W}
\end{array}\right]
$$

Figure 6. A Jacobian block of IMPES transfomation; 2P/undersaturated system 


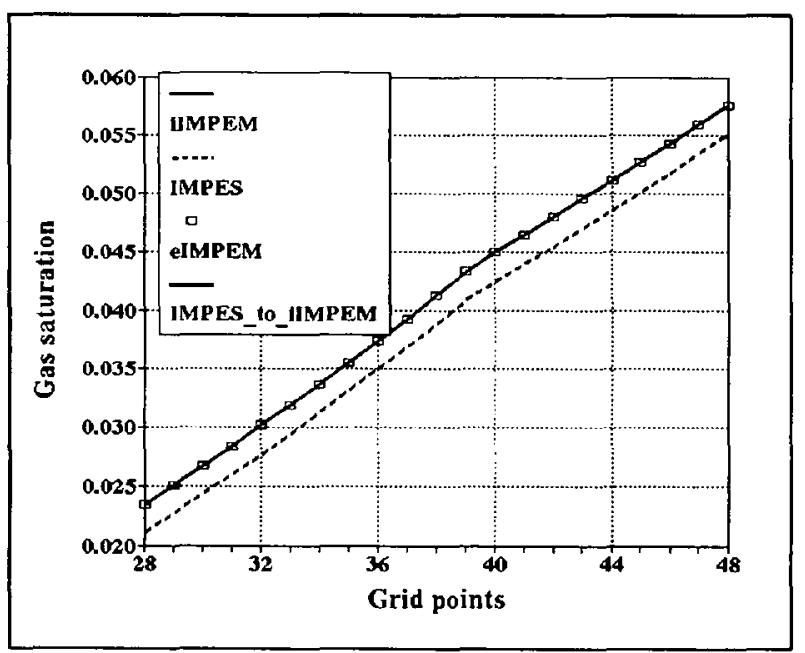

Figure 7. Gas saturations (no iteration)

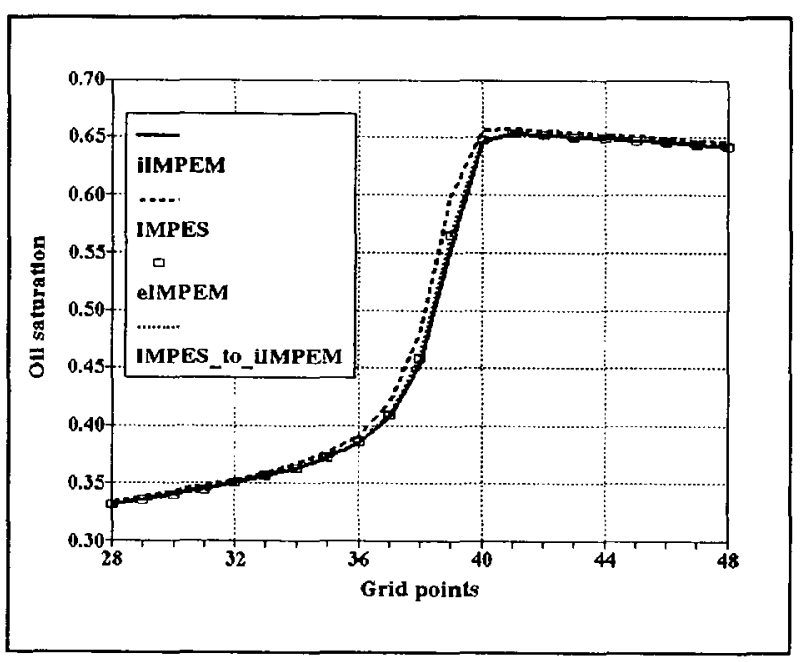

Figure 9. Oil saturations (no iteration)

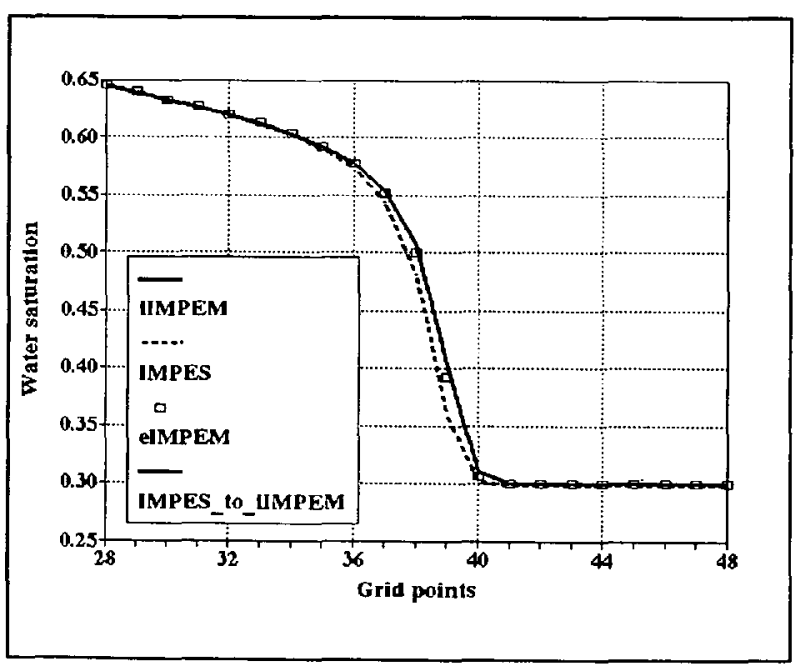

Figure 11. Water saturations (no iteration)

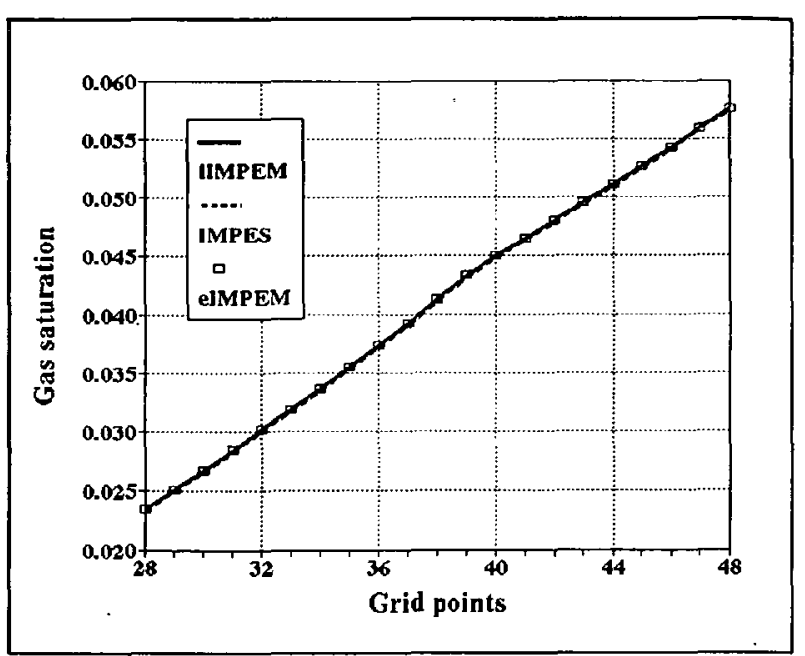

Figure 8. Gas saturations (one iteration)

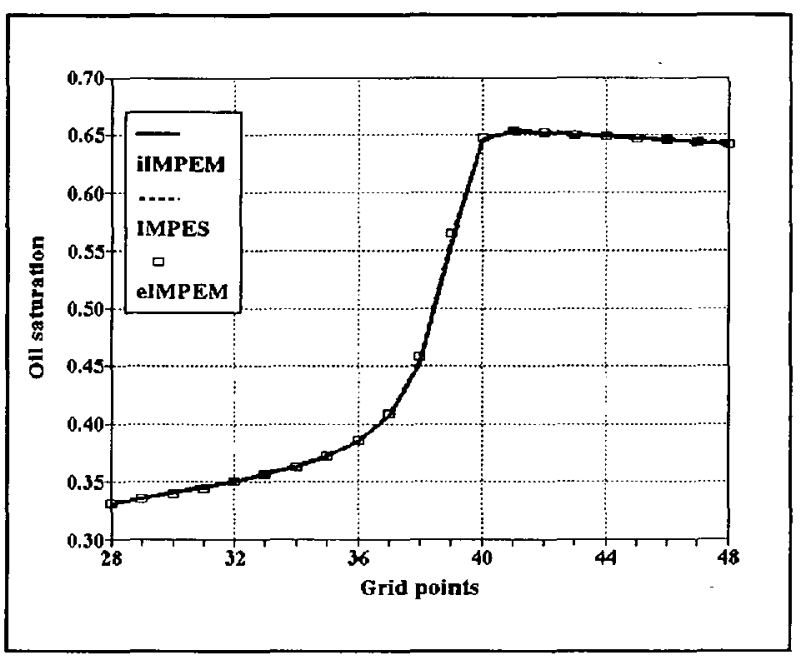

Figure 10. Oil saturations (one iteration)

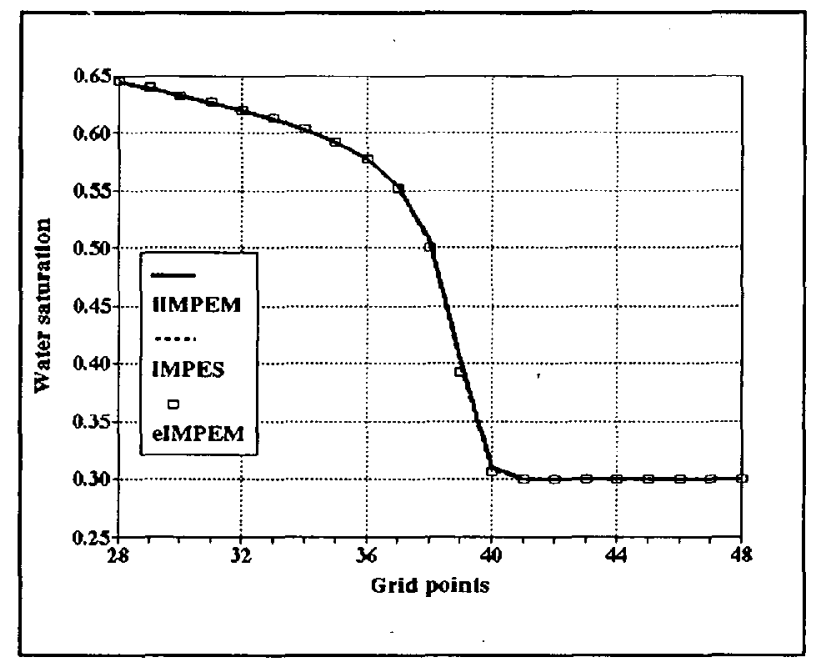

Figure 12. Water saturations (one iteration) 